\title{
THE PIERRE AUGER OBSERVATORY: SCIENCE PROSPECTS AND PERFORMANCE AT FIRST LIGHT
}

\author{
LUIS A. ANCHORDOQUI \\ [for the AUGER Collaboration] \\ Department of Physics \\ Northeastern University, \\ Boston MA 02115 \\ E-mail: l.anchordoqui@neu.edu
}

The Pierre Auger Observatory is a major international effort aiming at highstatistics study of highest energy cosmic rays. A general description of the experimental set-up and overall performance of the detector at first light are presented.

The Pierre Auger Observatory (PAO) is designed to study cosmic rays with energies above about $10^{10} \mathrm{GeV}$ with the aim of uncovering their origins and nature. ${ }^{1}$ Such events are too rare to be directly detected, but the direction, energy, and to some extent the chemical composition of the primary particle can be inferred from the cascade of secondary particles induced when the primary impinges on the upper atmosphere. ${ }^{2}$ These cascades, or air showers, have been studied in the past by measuring the nitrogen fluorescence they produce in the atmosphere ${ }^{3}$ or by directly sampling shower particles at ground level. ${ }^{4}$ The PAO is a "hybrid" detector, exploiting both of these methods by employing an array of water Cerenkov detectors overlooked by fluorescence telescopes; on clear, dark nights air showers are simultaneously observed by both types of detectors, facilitating powerful reconstruction methods and control of the systematic errors which have plagued cosmic ray experiments to date. Additionally, since the center-ofmass energy in the collision of the primary with the atmosphere is above about $100 \mathrm{TeV}$ (i.e., exceeding the contemporary and forthcoming collider reach by 2 orders of magnitude), PAO will also be capable of probing new physics beyond the electroweak scale. ${ }^{5}$

The Observatory will be covering two sites, in the Southern (Pampa Amarilla) and Northern hemispheres. Each site consist of 1600 stations 
spaced $1.5 \mathrm{~km}$ apart from each other, with 4 fluorescence eyes placed on the boundaries of the surface array. The energy threshold is defined by the $1.5 \mathrm{~km}$ spacing of the detector stations: a $10^{10} \mathrm{GeV}$ vertical shower will hit on average 6 stations which is enough to fully reconstruct the extensive air shower. The installation of the Southern site is now well underway, with detectors looking at the yet poorly covered part of the sky in which the direction of the center of the Milky Way is visible.

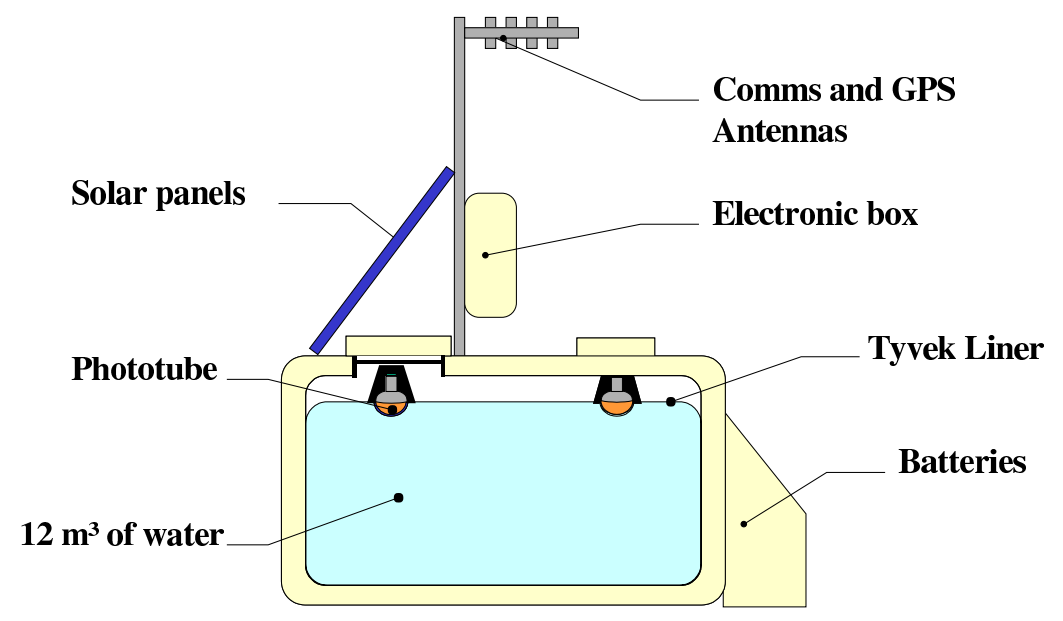

Figure 1. Schematic view of a water Čerenkov detector.

Each ground-based detector is a cylindrical opaque tank of $3.6 \mathrm{~m}$ diameter and $1.55 \mathrm{~m}$ high, where particles produce light by Čerenkov radiation, see Fig. 1. The filtered water is contained in a bag which diffusely reflects the light collected by three photomultipliers (PMT's) installed on the top. The large diameter PMT's $(\approx 20 \mathrm{~cm})$ are mounted facing down and look at the water through sealed polyethylene windows that are integral part of the internal liner.

Due to the size of the array, the detectors have to be able to function independently. The stations operate on battery-backed solar power and time synchronisation relies on a comercial Global Positioning System (GPS) receiver. ${ }^{6}$ A specially designed wireless LAN radio system is used to provide communication between the surface detectors and the central station. ${ }^{7}$ Each tank forms an autonomous unit, recording signals from the ambient cosmic ray flux, independent of the signals registered by any other tank in the surface detector array. A combination of signals clustered in space and 
time is used to identify a shower.

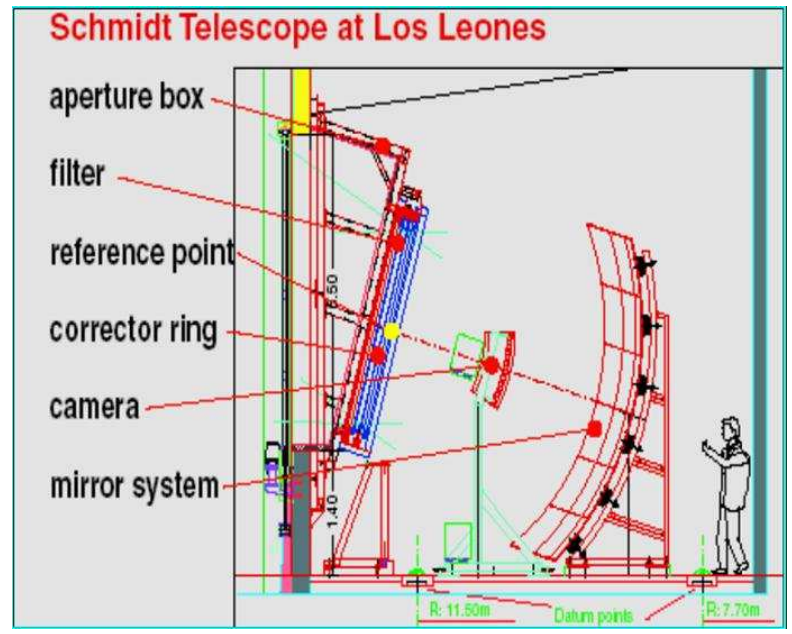

Figure 2. Schematic view of a fluorescence telescope at Los Leones. From left to right can be seen the aperture system, the photomultiplier camera and the spherical mirror.

A single fluorescence detector unit (or eye) comprises 6 telescopes, each located in independent bays, overlooking separate volumes of air. A schematic cross-sectional view of one fluorescence telescope is shown in Fig. 2. A circular diaphragm, positioned at the centre of curvature of the spherical mirror, defines the aperture of the Schmidt optical system. ${ }^{8}$ Ultraviolet transmitting filters are installed in the entrance aperture. Just inside the ultra-violet filter is a ring of (Schmidt) corrector elements. Light is focused by a large $3.5 \mathrm{~m} \times 3.5 \mathrm{~m}$ spherical mirror onto a 440 PMT camera, which accommodates the $30^{\circ}$ azimuth $\times 28.6^{\circ}$ elevation field of view. Each camera pixel has a field of view of approximately $1.5^{\circ}$.

In the hybrid mode ( $\sim 10 \%$ of the time) the detector is expected to have energy resolution of $13 \%(1 \sigma)$ at $10^{9} \mathrm{GeV}$ improving to $5.5 \%$ at $10^{11} \mathrm{GeV}$, and an angular resolution of about $0.5^{\circ}$. For the ground array alone these numbers become $10 \%$ and $1^{\circ}$, again for primary energy $>10^{11} \mathrm{GeV}$. Estimating event rates is a risky business because above $10^{11} \mathrm{GeV}$ the fluxes are essentially not known. ${ }^{9}$ However, for zenith angle less than $60^{\circ}$ the total aperture of the Southern surface array is $\sim 7350 \mathrm{~km}^{2}$ sr, and thus extrapolation from AGASA measurements ${ }^{9}$ implies that PAO should detect of the order of 2500 events above $10^{10} \mathrm{GeV}$ and of a 50 to 100 events above 
$10^{11} \mathrm{GeV}$ every year. Moreover, if events above 60 degrees can be analyzed effectively, the aperture will increase by about $50 \%$.

PAO also provides a promising way of detecting ultra-high energy neutrinos by looking for deeply-developing, large zenith angle $\left(>60^{\circ}\right)$ or horizontal air showers. ${ }^{10}$ At these large angles, hadronic showers have traversed the equivalent of several times the depth of the vertical atmosphere and their electromagnetic component has extinguished far away from the detector. Only very high energy core-produced muons survive past 2 equivalent vertical atmospheres. Therefore, the shape of a hadronic (background in this case) shower front is very flat and very prompt in time. In contrast, a neutrino shower appears pretty much as a "normal" shower. It is therefore straightforward to distinguish neutrino induced events from background hadronic showers. Moreover, if full flavor mixing is confirmed, tau neutrinos could be as abundant as other species and so very low $\nu_{\tau}$ fluxes could be detected very efficiently by PAO's detectors by looking at the interaction in the Earth crust of quasi horizontal $\nu_{\tau}$ inducing a horizontal cascade at the detector. ${ }^{11}$

The first PAO site is now operational in Malargüe, Argentina, and is in the process of growing to its final size of $3000 \mathrm{~km}^{2}$. At the time of writing, 12 telescopes and about 400 water tanks were operational. The first analyses of data from the PAO are currently underway. Figure 3 shows the arrival directions of all events recorded from January to July 2004. The pixels have a size of 1.8 degrees and the map was smoothed with a Gaussian beam of 5 degrees. ${ }^{12}$ On 21 May 2004, one of the larger events recorded by the surface array triggered 34 stations. A preliminary estimate yields an energy $\sim 10^{11} \mathrm{GeV}$ and a zenith angle of about $60^{\circ}$. First physics results will be made public in the Summer of 2005 at the 29th International Cosmic Ray Conference.

\section{Acknowledgments}

I would like to thank all my collaborators in the Pierre Auger Observatory. Special thanks goes to Tere Dova, Jean-Christophe Hamilton, Antoine Letessier-Selvon, Tom McCauley, Tom Paul, Steve Reucroft, and John Swain for assistance in the preparation of this talk. This work has been partially supported by the US National Science Foundation (NSF), under grant No. PHY-0140407. 


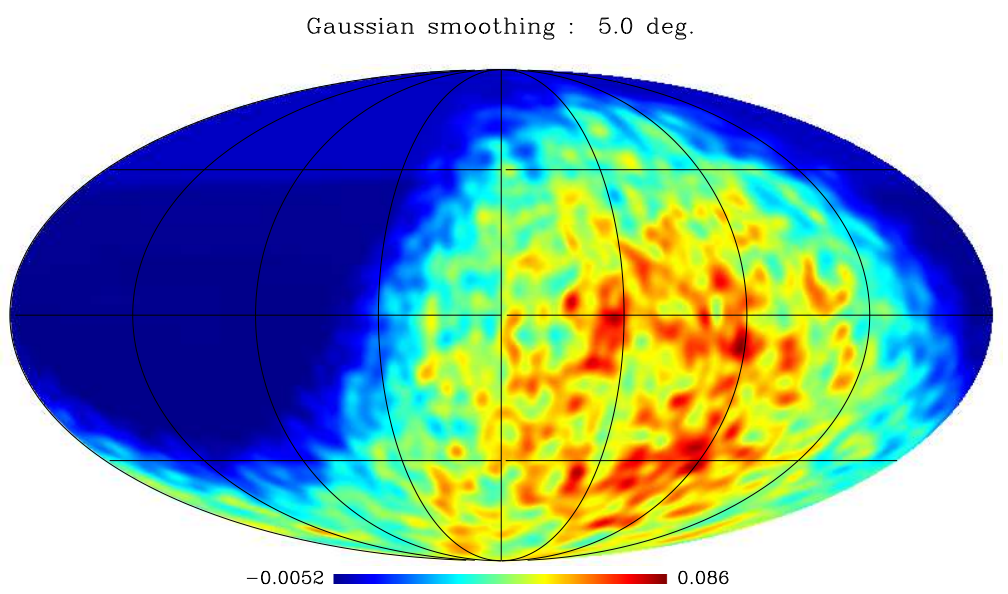

Figure 3. All event (from January to July 2004) skymap in Galactic coordinates. Units $x$ are related to the number of events per pixel $n$ according to: $n=330 x+1.716$.

\section{References}

1. J. Abraham et al. [AUGER Collaboration], Nucl. Instrum. Meth. A 523, 50 (2004).

2. L. Anchordoqui, T. Paul, S. Reucroft and J. Swain, Int. J. Mod. Phys. A 18, $2229(2003)$.

3. R. M. Baltrusaitis et al., Nucl. Instrum. Meth. A 240, 410 (1985).

4. N. Chiba et al., Nucl. Instrum. Meth. A 311, 338 (1992).

5. L. A. Anchordoqui, arXiv:hep-ph/0306078. See also, L. Anchordoqui, H. Goldberg and C. Nunez, arXiv:hep-ph/0408284; L. Smolin, in these Proceedings.

6. C. L. Pryke and J. Lloyd-Evans, Nucl. Instrum. Meth. A 354, 560 (1995).

7. P. D. J.Clark and D. Nitz, Proc. of 27th International Cosmic Ray Conference, Copernicus Gesellschaft, 765 (2001).

8. A. Cordero et al., GAP-1996-039.

9. M. Takeda et al. [AGASA Collaboration], Astropart. Phys. 19, 447 (2003); R. U. Abbasi et al. [HiRes Collaboration], Phys. Rev. Lett. 92, 151101 (2004).

10. K. S. Capelle, J. W. Cronin, G. Parente and E. Zas, Astropart. Phys. 8, 321 (1998).

11. X. Bertou, P. Billoir, O. Deligny, C. Lachaud and A. Letessier-Selvon, Astropart. Phys. 17, 183 (2002); J. L. Feng, P. Fisher, F. Wilczek and T. M. Yu, Phys. Rev. Lett. 88, 161102 (2002).

12. E. Armengaud et al., GAP-2003-105.

The references to internal reports written by the AUGER Collaboration (GAP notes) are accesible via www . auger.org/admin/GAP_NOTES. 